\title{
Dynkin 型路代数倾斜模与完全切片模*
}

\author{
王敏雄 ${ }^{1}$ 林亚南 ${ }^{2}$ \\ (1. 华侨大学数学系, 泉州 $362011 ; 2$. 厦门大学数学系, 厦门 361005)
}

摘要 设 $T$ 是 Dynkin 型路代数的倾斜模, 若 $T$ 的不可分解直和项在不同的 $\tau$ 轨道上, 证明了 $T$ 为完全切片模.

关键词 倾斜模 路代数 切片模 hammock

\section{1 主要结论}

本文总约定代数 $A$ 是代数闭域 $k$ 上的 Dynkin 型路代数 $k \Delta$. 模总指有限生成 右 $A$ - 模. 本文不区分模和它的同构类, 不区分不可分解模和 Auslander-Reiten 箭 图对应的点. 映射 $f: x \rightarrow y$ 与 $g: y \rightarrow z$ 的合成记为 $f g$.

设 $\Gamma_{A}$ 是 Dynkin 型路代数 $A$ 的 Auslander-Reiten 箭图 (AR- 箭图), $\tau$ 是 AuslanderReiten 变换. 设 $\mathcal{U}$ 是 $\Gamma_{A}$ 的连通子图且与每个 $\tau$ 轨道恰好相交于一点, 则称 $\mathcal{U}$ 是一 个完全切片 (complete slice), $\mathcal{U}$ 中点所对应的不可分解模的直和 $U=\bigoplus_{U(a) \in \mathcal{U}} U(a)$ 称为完全切片模 (complete slice module) ${ }^{[1]}$.

定义 1 $^{[2]}$ 一个 $A$ - 模 $T_{A}$ 称为倾斜模, 如果满足:

(1) $\operatorname{pd} T_{A} \leqslant 1$;

(2) $\operatorname{Ext}_{A}^{1}\left(T_{A}, T_{A}\right)=0$;

(3) 存在正合列 $0 \rightarrow A_{A} \rightarrow T_{A}^{\prime} \rightarrow T_{A}^{\prime \prime} \rightarrow 0$, 其中 $T_{A}^{\prime}, T_{A}^{\prime \prime}$ 是 $T$ 的直和项的直 和.

从 20 世纪 70 年代开始, 倾斜模以及倾斜代数得到深入的研究, 它成为有限 维代数表示理论的核心部分之一和最重要的技巧之一. Dynkin 型路代数的完全 切片模是一个倾斜模. 倾斜模是完全切片模当且仅当它是可分倾斜模 ${ }^{[3]}$. 本文的 主要结果是下面

定理 设 $A$ 是 Dynkin 型路代数, $T=\bigoplus_{i=1}^{n} T_{i}$ 是倾斜 $A$ - 模, 其中 $T_{i}(1 \leqslant$ $i \leqslant n)$ 是不可分解直和项. 如果 $T_{i}(1 \leqslant i \leqslant n)$ 分布在不同的 $\tau$ 轨道上, 则 $T$ 是 一个完全切片模.

2003-05-28 收稿, 2003-10-22 收修改稿

* 国家自然科学基金 (批准号: 10371101) 和华侨大学自然科学基金资助项目 
要证明这一结果, 只要说明倾斜模 $T$ 的全部直和项 $T_{1}, T_{2}, \cdots, T_{n}$ 作成 $\Gamma_{A}$ 的 一个连通子图, 这是因为这里的 $n$ 恰是单 $A$ - 模的个数, 从而恰是 $\Gamma_{A}$ 中 $\tau$ 轨道 的个数. 定理的证明依赖于组合分析, 依赖于对 $Z \Delta$ 上的完全切片与 hammock ${ }^{[4]}$ 的分析.

\section{$2 Z \Delta$ 上的完全切片与 hammock}

给定一个 Dynkin 型有向箭图 $\Delta=\left(\Delta_{0}, \Delta_{1}\right)$, 可以构造变换图 $Z \Delta$, 它的点 是 $(a, x), a \in Z, x \in \Delta_{0}$, 对 $\Delta_{1}$ 中的箭 $\alpha: x \mapsto y$ 和任意 $a \in Z$, 我们定义 $Z \Delta$ 中的箭 $(a, x) \mapsto(a, y)$ 和 $(a, y) \mapsto(a+1, x)$. 最后, $Z \Delta$ 的变换 $\tau$ 的定义为 $\tau:(a, x) \mapsto(a-1, x)$. $(a, x)$ 所在的 $\tau$ - 轨道为 $\{(b, x), b \in Z\}$.

设 $x, y \in Z \Delta$, 称 $x \leqslant y$, 如果 $Z \Delta$ 中存在从 $x$ 到 $y$ 的路. 称 $Z \Delta$ 的一个连通子 图 $\mathcal{S}$ 是一个完全切片, 如果它与每个 $\tau$ 轨道恰好相交于一点. 设 $\mathcal{S}, \mathcal{T}$ 是 $Z \Delta$ 上 两个完全切片, $y \in Z \Delta$. 称 $\mathcal{S} \leqslant y$, 如果存在点 $x \in \mathcal{S}$, 使得 $x \leqslant y$; 称 $y \leqslant \mathcal{S}$, 如果 存在点 $x \in \mathcal{S}$, 使得 $y \leqslant x$. 易见 $y \leqslant \mathcal{S}$ 且 $\mathcal{S} \leqslant y$ 当且仅当 $y \in \mathcal{S}$. 称 $\mathcal{S} \leqslant \mathcal{T}$, 若对任 意 $s \in \mathcal{S}$ 有 $s \leqslant \mathcal{T}$, 且对任意 $t \in \mathcal{T}$ 有 $\mathcal{S} \leqslant t$. 当 $u \leqslant v$ 时, $(v, t)$ 称为 $(u, t)$ 的 $\tau$-后 继元. $Z \Delta$ 中一条路 $\alpha_{1} \alpha_{2} \cdots \alpha_{t}$ 称为 section 路, 如果 $\tau e\left(\alpha_{i+1}\right) \neq s\left(\alpha_{i}\right)(1 \leqslant i<t)$, 这里 $e\left(\alpha_{i+1}\right)$ 是 $\alpha_{i+1}$ 的终点, $s\left(\alpha_{i}\right)$ 是 $\alpha_{i}$ 的起点. 给定点 $x \in Z \Delta$, 定义

(1) $\mathcal{S}(\rightarrow x)=\{y \in Z \Delta \mid y \leqslant x$, 且从 $y$ 到 $x$ 的任意路都是 section $\}$;

(2) $\mathcal{S}(x \rightarrow)=\{z \in Z \Delta \mid x \leqslant z$, 且任意从 $x$ 到 $z$ 的路都是 section $\}$.

易见 $\mathcal{S}(\rightarrow x)$ 与 $\mathcal{S}(x \rightarrow)$ 都是完全切片. 设 $\mathcal{T}$ 是 $Z \Delta$ 的一个完全切片, $x \in \mathcal{T}$, 则对于任意 $y \in \mathcal{T}$, 有 $\mathcal{S}(\rightarrow x) \leqslant y \leqslant \mathcal{S}(x \rightarrow)$.

当 $A=k \Delta$ 为 Dynkin 型的路代数时, 将 $\Gamma_{A}$ 视为 $Z \Delta$ 的子图. $\Gamma_{A}$ 的投射 点构成一个完全切片, 记为 $\mathcal{S}(P)$; 所有入射点构成一个完全切片, 记为 $\mathcal{S}(Q)$. $\Gamma_{A}=\{z \in Z \Delta \mid \mathcal{S}(P) \leqslant z \leqslant \mathcal{S}(Q)\}$.

引理 1 设 $A=k \Delta$ 为 Dynkin 型路代数, $\Gamma_{A}$ 是 $Z \Delta$ 子图, $x \in Z \Delta$, 则

(1) $\left\{z \in Z \Delta \mid \operatorname{Hom}_{k(Z \Delta)}(x, z) \neq 0\right\}$ 是以 $x$ 为起点, $y$ 为终点的 hammock, 记 为 ${ }_{x} H_{y}$, 这里 $k(Z \Delta)$ 是 $Z \Delta$ 的 mesh 范畴;

进一步, 对于 ${ }_{x} H_{y}$,

(2) 如果 $y \in \Gamma_{A}$, 则任意的 $z \in \Gamma_{A}, \mathcal{S}(\rightarrow x) \leqslant z$;

(3) 如果 $x \in \Gamma_{A}$, 则任意的 $z \in \Gamma_{A}, z \leqslant \mathcal{S}(y \rightarrow)$;

(4) 如果 $x, y \in \Gamma_{A}$, 则 $x$ 是 $\Gamma_{A}$ 的投射点, $y$ 是 $\Gamma_{A}$ 的入射点, 对应的不可分 解投射模 $P$ 与不可分解入射模 $Q$ 具有性质 $\operatorname{top} P=\operatorname{soc} Q$.

证 (1) $\mathcal{S}(x \rightarrow)$ 是 $Z \Delta$ 的一个完全切片, 记为 $\Delta_{1}$, 则 $B=k \Delta_{1}$ 是与 $A$ 同型 的路代数, $Z \Delta=Z \Delta_{1}, \Gamma_{B}$ 嵌入 $Z \Delta$ 中, 则 ${ }_{x} H=\left\{z \in Z \Delta \mid \operatorname{Hom}_{k(Z \Delta)}(x, z) \neq 0\right\}$ $=\left\{z \in \Gamma_{B} \mid \operatorname{Hom}_{k\left(\Gamma_{B}\right)}(x, z) \neq 0\right\}$ 是以单投射 $B$ - 模 $x$ 为起点的 hammock.

(2) 设 $y \in \Gamma_{A}$, 记 $\Gamma_{A}$ 中 $y$ 所在 $\tau$ - 轨道的入射点为 $q$, 记 ${ }_{p} H_{q}$ 是以 $q$ 为终点 的 hammock, 其起点 $p$ 是 $\Gamma_{A}$ 的投射点. 若 $y=q$, 则 $x=p$. 若 $y \neq q$, 不妨设 
$q=\tau^{-l} y(l>0)$, 则 ${ }_{x} H_{y}$ 由 ${ }_{p} H_{q}$ 左平移 $\tau^{l}$ 次得到, $p=\tau^{-l} x$, 故 $x \notin \Gamma_{A}$, 则 $\mathcal{S}(\rightarrow x)$ 是 $\mathcal{S}(\rightarrow p)$ 左平移 $\tau^{l}$ 次的切片, 所以 $\mathcal{S}(\rightarrow x) \leqslant \mathcal{S}(\rightarrow p) \leqslant \mathcal{S}(P)$, 对任意 $z \in \Gamma_{A}, \mathcal{S}(P) \leqslant z$, 故有 $\mathcal{S}(\rightarrow x) \leqslant z$.

(3) 对偶于 (2) 的证明.

(4) 显然.

证毕.

\section{3 定理的证明}

\section{1 线性 $A_{n}$ 型的情形}

设 $A$ 为线性 $A_{n}$ 型 Dynkin 图的 $\Delta$ 路代数, 即

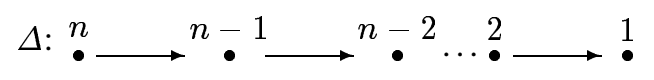

则 $A$ 的 AR- 箭图 $\Gamma_{A}$ 形如图 1 , 其中 $p_{i}$ 对应不可分解投射模 $P_{i}, 1 \leqslant i \leqslant n$.

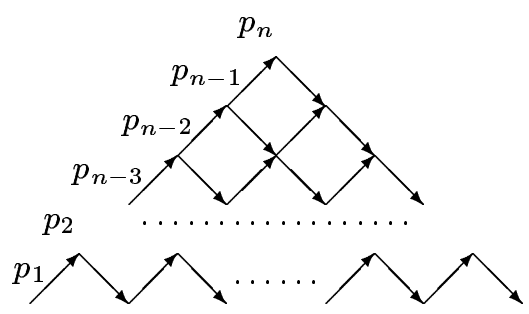

图 1 线性 $A_{n}$ 型路代数的 $\mathrm{AR}-$ 箭图

设 $T=\bigoplus_{i=1}^{n} T_{i}$ 是线性 $A_{n}$ 型路代数的倾斜模, $T_{i}(1 \leqslant i \leqslant n)$ 在不同的 $\tau$ 轨 道上. 设 $T_{i}$ 在 $\Gamma_{A}$ 对应的点是 $t_{i}(1 \leqslant i \leqslant n), t_{i}=\tau^{-r_{i}} p_{i}\left(0 \leqslant r_{i} \leqslant n-i, 1 \leqslant i \leqslant n\right)$. 显然, $t_{n}=p_{n}$ 且 $t_{n-1}=p_{n-1}$ 或 $\tau^{-1} p_{n-1}$, 所以 $t_{n}$ 和 $t_{n-1}$ 相连. 当 $t_{n-1}=p_{n-1}$ 时, 由 $0=\operatorname{Ext}_{A}^{1}\left(T_{i}, T_{n-1}\right)=D \operatorname{Hom}_{A}\left(\tau^{-1} T_{n-1}, T_{i}\right)$ 知 $t_{i} \neq \tau^{-(n-i)} p_{i}(1 \leqslant i \leqslant n-1)$, 故 $t_{i}(1 \leqslant i \leqslant n-1)$ 在一个与线性 $A_{n-1}$ 型的 AR- 箭图同构的子图中, 由归 纳假设知 $t_{i}$ 与 $t_{i-1}$ 是邻点 $(1 \leqslant i \leqslant n-1)$. 当 $t_{n-1}=\tau^{-1} p_{n-1}$ 时, 由 $0=$ $\operatorname{Ext}_{A}^{1}\left(T_{n-1}, T_{j}\right)=D \operatorname{Hom}_{A}\left(T_{j}, \tau T_{n-1}\right)$ 知 $t_{i} \neq p_{i}(1 \leqslant i \leqslant n-1)$. 同理知 $t_{i}$ 与 $t_{i-1}$ 是邻点 $(1 \leqslant i \leqslant n-1)$, 即 $T=\bigoplus_{i=1}^{n} T_{i}$ 是完全倾斜模. 证毕.

\section{$3.2 \boldsymbol{A}_{2 m+1}$ 型情形}

不妨考虑变换图 $Z \Delta_{A_{2 m+1}}$, 这里 $\Delta_{A_{2 m+1}}$ 为: $2 m \leftarrow 2 m-2 \leftarrow \cdots \leftarrow 2 \leftarrow$ $1 \rightarrow 3 \rightarrow \cdots \rightarrow 2 m-1 \rightarrow 2 m+1$ (图 2 是 $m=5$ 的情形).

由 hammock 的定义, 可直接验证下列结论:

引理 2 在 $Z \Delta_{A_{2 m+1}}$ 中, 


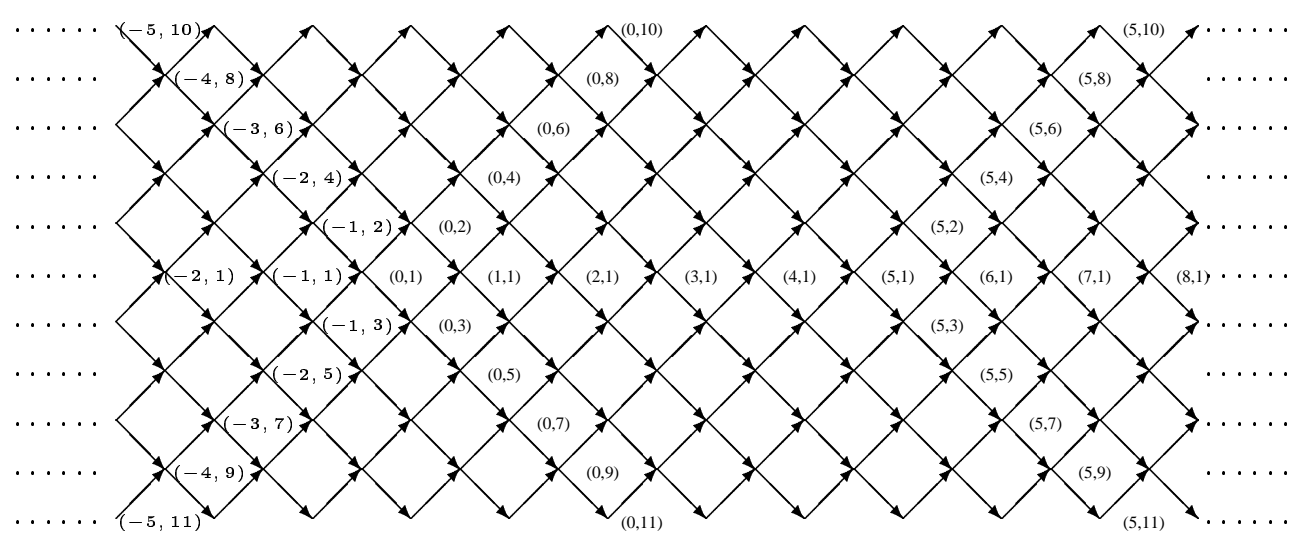

图 $2 Z \Delta_{A_{11}}$

(1) 设 ${ }_{x} H_{y}$ 是 hammock, 则 ${ }_{x} H_{y}=\{z \mid \mathcal{S}(x \rightarrow) \leqslant z \leqslant \mathcal{S}(\rightarrow y)\}$;

(2) 以 $(s, 1)$ 为起点的 hammock 的终点为 $(s+m, 1)$;

(3) 当 $t$ 为不小于 3 的奇数时, 以 $(s, t)$ 为起点的 hammock 的终点为 $(s+m, t-$ $1)$;

(4) 当 $t$ 为偶数时, 以 $(s, t)$ 为起点的 hammock 的终点为 $(s+m, t+1)$.

引理 3 设 $\Gamma_{A}$ 是 $A_{2 m+1}$ 型的路代数的 AR- 箭图, $\Gamma_{A}$ 嵌入 $Z \Delta_{A_{2 m+1}}$. 设 $(s, 1)$ 是 $\Gamma_{A}$ 中某个点的 $\tau$ - 后继元, $(s, t) \in \Gamma_{A}$, 则

(1) 当 $t$ 为偶数时, $(s, 1) \rightarrow(s, 2) \rightarrow(s, 4) \rightarrow \cdots \rightarrow(s, t)$ 是 $\Gamma_{A}$ 的子图;

(2) 当 $t$ 为奇数时, $(s, 1) \rightarrow(s, 3) \rightarrow(s, 5) \rightarrow \cdots \rightarrow(s, t)$ 是 $\Gamma_{A}$ 的子图.

证 设 $t$ 为偶数. 因 $(s, 1)$ 是 $\Gamma_{A}$ 中某个点的 $\tau$-后继元, 设 $\Gamma_{A}$ 在 $(s, i)$ 的 $\tau$ - 轨道上的投射点为 $p_{i}$, 则 $(s, 1)$ 是 $p_{1}$ 的 $\tau$ - 后继元. 所以 $\mathcal{S}\left(\rightarrow p_{1}\right) \leqslant \mathcal{S}(P) \leqslant$ $\mathcal{S}((s, 1) \rightarrow)$, 从而 $\mathcal{S}(P) \leqslant(s, i)(i=1,2,4, \cdots, t)$. 同理, 考虑 $q_{t}$ 是 $(s, t)$ 的 $\tau$ - 后 继元, 可得 $(s, i) \leqslant \mathcal{S}(Q)(i=1,2,4, \cdots, t)$, 所以 $(s, i) \in \Gamma_{A}$. 同理可证 $t$ 为奇数情 形. 证毕.

将 $\Gamma_{A}$ 作为 $Z \Delta_{A_{2 m+1}}$ 的子图. 不妨设 $T_{1}$ 对应于点 $(0,1), T_{i}$ 对应的点 $t_{i}$ 在 $(0, i)$ 所在的 $\tau$ - 轨道上. 由 AR- 公式 ${ }^{[1]}$ 与倾斜模的定义, $0=\operatorname{Ext}_{A}^{1}\left(T_{i}, T_{1}\right)=$ $D \operatorname{Hom}_{A}\left(\tau^{-1} T_{1}, T_{i}\right) . \tau^{-1} T_{1}$ 的对应点是 $(1,1),{ }_{(1,1)} H$ 的终点为 $(m+1,1)$, 所以 $t_{i} \notin$ $\{z \mid \mathcal{S}((1,1) \rightarrow) \leqslant z \leqslant \mathcal{S}(\rightarrow(m+1,1))\}(2 \leqslant i \leqslant 2 m+1)$. 又因 $(0,1) \in \Gamma_{A},={ }_{(0,1)} H$ 的终点为 $(m, 1)$, 由引理 $1, t_{i} \leqslant \mathcal{S}((m, 1) \rightarrow)$. 对偶地, 有 $t_{i} \notin\{z \mid \mathcal{S}((-m-1,1)$ $\rightarrow) \leqslant z \leqslant \mathcal{S}(\rightarrow(-1,1))\}$, 并且 $\mathcal{S}(\rightarrow(-m, 1)) \leqslant t_{i}$.

记 $\mathcal{W}(0,1)=\{z \mid \mathcal{S}(\rightarrow(0,1)) \leqslant z \leqslant \mathcal{S}((0,1) \rightarrow)\}, \mathcal{W}^{(m, 4)}=\{z \mid \mathcal{S}((2,2 m)$

$\rightarrow) \leqslant z \leqslant \mathcal{S}(\rightarrow(m, 2 m))\}, \mathcal{W}_{(m, 5)}=\{z \mid \mathcal{S}((2,2 m+1) \rightarrow) \leqslant z \leqslant \mathcal{S}(\rightarrow(m, 2 m+$ $1))\}, \mathcal{W}^{(-m-2,4)}=\{z \mid \mathcal{S}((-2 m, 2 m) \rightarrow) \leqslant z \leqslant \mathcal{S}(\rightarrow(-m-2,2 m))\}, \mathcal{W}_{(-m-2,5)}=$ $\{z \mid \mathcal{S}((-2 m, 2 m+1) \rightarrow) \leqslant z \leqslant \mathcal{S}(\rightarrow(-m-2,2 m+1))\}$. 记 $\overline{\mathcal{W}}=\mathcal{W}(0,1) \cup \mathcal{W}^{(m, 4)}$ $\cup \mathcal{W}_{(m, 5)} \cup \mathcal{W}^{(-m-2,4)} \cup \mathcal{W}_{(-m-2,5)}$, 从而 $t_{i} \in \overline{\mathcal{W}}(1 \leqslant i \leqslant n)$. 
为证明定理, 只要证明: 若 $t_{i}$ 与 $t_{j}$ 的 $\tau$ - 轨道相邻, 则 $t_{i}$ 与 $t_{j}$ 是 $Z \Delta_{A_{2 m+1}}$ 中 的邻点. 对 $i$ 作归纳.

因为 $\overline{\mathcal{W}}$ 中在 $t_{2}$ 所在的轨道的点只有 $(-1,2)$ 与 $(0,2)$, 它们均是 $t_{1}$ 的邻 点, 同理 $t_{3}$ 是 $t_{1}$ 的邻点. $\overline{\mathcal{W}}$ 中 $t_{4}$ 所在的轨道有点 $(0,4),(-1,4),(-2,4),(m, 4)$ 及 $(-m-2,4)$. 假设 $t_{4}=(m, 4)$, 则 $t_{1}$ 的 $\tau$ - 后继元 $(m, 1) \in \Gamma_{A}$. 由引理 3 , $(m, 1),(m, 2),(m, 4) \in \Gamma_{A}$. 进而根据引理 1 之 (4) 知 $(0,1)$ 是 $\Gamma_{A}$ 的投射点, $(m, 1)$ 是 $\Gamma_{A}$ 的入射点, 因而 $(m, 1),(m, 2),(m, 4)$ 均为 $\Gamma_{A}$ 的入射点. 相应地由引理 2 知 $(0,1),(0,3),(0,5)$ 是 $\Gamma_{A}$ 的投射点, 所以 $t_{3}=(0,3)$. 因为 $0=\operatorname{Ext}_{A}^{1}\left(T_{4}, T_{3}\right)=$ $D \operatorname{Hom}_{A}\left(T_{3}, \tau T_{4}\right), \tau t_{4}=(m-1,4)$, 由引理 2, 以 $\tau t_{4}$ 为终点的 hammock 的起点为 $(-1,5)$. 又 $(0,3) \in{ }_{(-1,5)} H_{\tau t_{4}}$, 故 $\operatorname{Hom}_{A}\left(T_{3}, \tau T_{4}\right) \neq 0$, 矛盾, 这样 $t_{4} \neq(m, 4)$. 对 偶地讨论可得 $t_{4} \neq(-m-2,4)$, 所以 $t_{4} \in \mathcal{W}^{(0,1)}$. 根据线性 $A_{n}$ 型情形主定理的 证明, 知 $t_{4}$ 是 $t_{2}$ 的邻点. 同理 $t_{5}$ 是 $t_{3}$ 的邻点.

假设当 $i, j \leqslant 2 k-1$ 时, 若 $t_{i}$ 与 $t_{j}$ 的 $\tau$ - 轨道相邻, 则 $t_{i}$ 与 $t_{j}$ 是邻点. 考 虑 $t_{2 k}$. 如果 $t_{2 k}=(s, 2 k) \in \mathcal{W}^{(m, 4)}$, 由引理 $3,(s, 1),(s, 2),(s, 4), \cdots,(s, 2 k) \in \Gamma_{A}$, 由引理 1 之 (2), $\Gamma_{A}$ 中的 $(s-m, i)$ 所在轨道的点是 $(s-m, i)$ 的 $\tau$ - 后继元 $(i=$ $1,3,5, \cdots, 2 k+1)$. 特别地, $t_{2 k-1}$ 是 $(s-m, 2 k-1)$ 的 $\tau$ - 后继元. 根据 AR-公 式, 有 $0=\operatorname{Ext}_{A}^{1}\left(T_{2 k}, T_{i}\right)=D \operatorname{Hom}_{A}\left(T_{i}, \tau T_{2 k}\right), \tau t_{2 k}=(s-1,2 k)$, 所以 $t_{i}$ 不能在 以 $\tau t_{2 k}$ 为终点的 hammock 中, 而这个 hammock 起点是 $(s-m-1,2 k+1)$, 所以 $t_{i} \notin \mathcal{S}((s-m-1,2 k+1) \rightarrow)$. 又 $(s-m, 2 k-1) \in \mathcal{S}((s-m-1,2 k+1) \rightarrow)$, 所以 $t_{2 k-1} \neq(s-m, 2 k-1)$. 而 $t_{2 k-1}$ 是 $(s-m, 2 k-1)$ 的 $\tau$ - 后继元, 我们在 $\mathcal{W}_{(0,1)}$ 中 考察, 存在 $j \in\{3,5, \cdots, 2 k-3\}$, 使得 $t_{j} \in \mathcal{S}((s-m-1,2 k+1) \rightarrow)$ 上, 矛盾.

所以 $t_{2 k} \notin \mathcal{W}^{(m, 4)}$.

对偶地讨论知 $t_{2 k} \notin \mathcal{W}^{(-m-2,4)}$, 因而 $t_{2 k} \in \mathcal{W}(0,1)$. 类似线性 $A_{n}$ 型情形主 定理的证明, 知 $t_{2 k}$ 与 $t_{2 k-2}$ 是邻点.

同理可知, 当 $i, j \leqslant 2 k$ 时命题成立, 故当 $i, j \leqslant 2 k+1$ 时命题也成立. 由数 学归纳法, 当 $\Delta$ 是 $A_{2 m+1}$ 型的情形时, 定理成立.

\section{$3.3 \boldsymbol{A}_{2 m}$ 型情形}

不妨考虑变换图 $Z \Delta_{A_{2 m}}$, 这里 $\Delta_{A_{2 m}}$ 为: $2 m \leftarrow 2 m-2 \leftarrow \cdots \leftarrow 2 \leftarrow 1 \rightarrow$ $3 \rightarrow \cdots \rightarrow 2 m-3 \rightarrow 2 m-1$ (图 3 是 $m=5$ 的情形).

引理 4 在 $Z \Delta_{A_{2 m}}$ 中,

(1) ${ }_{x} H_{y}$ 是 hammock, 则 ${ }_{x} H_{y}=\{z \mid \mathcal{S}(x \rightarrow) \leqslant z \leqslant \mathcal{S}(\rightarrow y)\}$;

(2) 以 $(s, 1)$ 为起点的 hammock 的终点为 $(s+m-1,2)$;

(3) 当 $t$ 为不小于 3 的奇数时, 以 $(s, t)$ 为起点的 hammock 的终点为 $(s+m-$ $1, t+1)$;

(4) 当 $t$ 为偶数时, 以 $(s, t)$ 为起点的 hammock 的终点为 $(s+m, t-1)$.

引理 5 设 $\Gamma_{A}$ 是 $A_{2 m}$ 型的路代数的 $\mathrm{AR}$ - 箭图, $\Gamma_{A}$ 嵌入 $Z \Delta_{A_{2 m}}$. 设 $(s, 1)$ 是 $\Gamma_{A}$ 中某个点的 $\tau$ - 后继元, $(s, t) \in \Gamma_{A}$, 则 


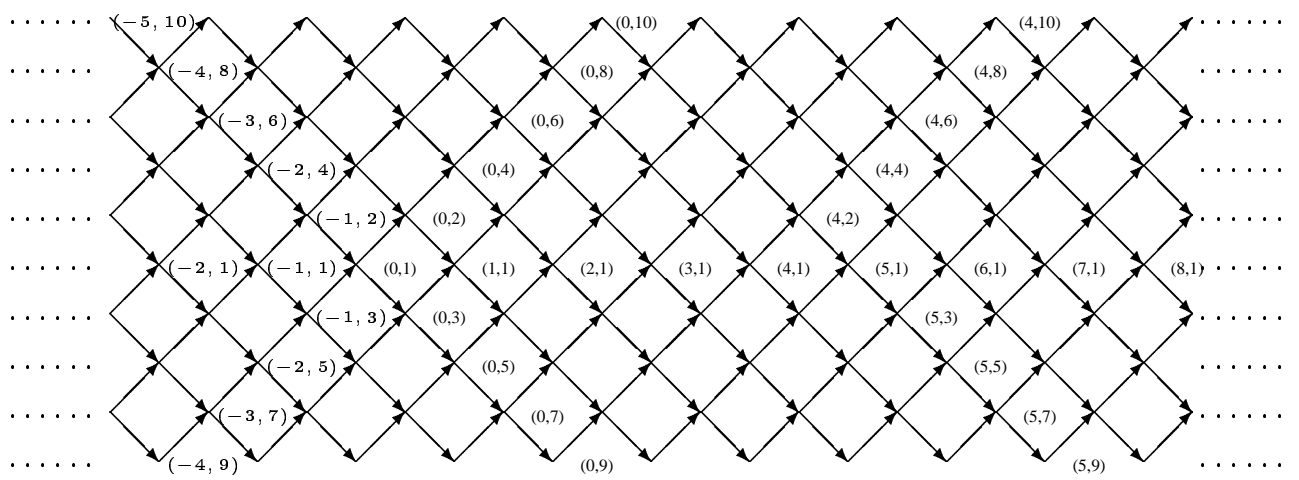

图 $3 Z \Delta_{A_{10}}$

(1) 当 $t$ 为偶数时, $(s, 1) \rightarrow(s, 2) \rightarrow(s, 4) \rightarrow \cdots \rightarrow(s, t)$ 是 $\Gamma_{A}$ 的子图;

(2) 当 $t$ 为奇数时, $(s, 1) \rightarrow(s, 3) \rightarrow(s, 5) \rightarrow \cdots \rightarrow(s, t)$ 是 $\Gamma_{A}$ 的子图.

将 $\Gamma_{A}$ 作为 $Z \Delta_{A_{2 m}}$ 的子图. 不妨设 $T_{1}$ 对应 $(0,1), T_{i}$ 对应的点 $t_{i}$ 在 $(0, i)$ 所在 的 $\tau$ - 轨道上. 记 $\mathcal{W}(0,1)=\{z \mid \mathcal{S}(\rightarrow(0,1)) \leqslant z \leqslant \mathcal{S}((0,1) \rightarrow)\}, \mathcal{W}^{(m-1,6)}=\{z \mid$ $\mathcal{S}((2,2 m) \rightarrow) \leqslant z \leqslant \mathcal{S}(\rightarrow(m-1,2 m))\}, \mathcal{W}_{(m, 3)}=\{z \mid \mathcal{S}((2,2 m-1) \rightarrow) \leqslant z \leqslant$ $\mathcal{S}(\rightarrow(m, 2 m-1))\}, \mathcal{W}^{(-m-2,6)}=\{z \mid \mathcal{S}((-2 m+1,2 m) \rightarrow) \leqslant z \leqslant \mathcal{S}(\rightarrow(-m-$ $2,2 m))\}, \mathcal{W}_{(-m-1,3)}=\{z \mid \mathcal{S}((-2 m+1,2 m-1) \rightarrow) \leqslant z \leqslant \mathcal{S}(\rightarrow(-m-1,2 m-1))\}$. 记 $\overline{\mathcal{W}}=\mathcal{W}(0,1) \cup \mathcal{W}^{(m-1,6)} \cup \mathcal{W}_{(m, 3)} \cup \mathcal{W}^{(-m-2,6)} \cup \mathcal{W}_{(-m-1,3)}$. 这样与 $A_{2 m+1}$ 中的证明类似, 可得到 $t_{i} \in \overline{\mathcal{W}}(1 \leqslant i \leqslant n)$.

下面证明如果 $t_{i}$ 与 $t_{j}$ 的 $\tau$ - 轨道相邻, 则 $t_{i}$ 与 $t_{j}$ 是 $Z \Delta_{A_{2 m}}$ 中的邻点. 对 $i$ 作归纳.

因为在 $\overline{\mathcal{W}}$ 中 $(0,2)$ 所在的轨道的点只有 $(-1,2)$ 与 $(0,2),(0,4)$ 所在的轨道的 点只有 $(0,4),(-1,4),(-2,4)$. 易证 $t_{3} \in \mathcal{W}_{(0,1)}$. 这样可得当 $1 \leqslant i \leqslant 4$ 时, 若 $t_{i}$ 与 $t_{j}$ 的 $\tau$ - 轨道相邻, 则 $t_{i}$ 与 $t_{j}$ 是邻点.

假设当 $i, j \leqslant 2 k$ 时, 若 $t_{i}$ 与 $t_{j}$ 的 $\tau$ - 轨道相邻, 则 $t_{i}$ 与 $t_{j}$ 是邻点, 考虑 $t_{2 k+1}$. 如果 $t_{2 k+1}=(s, 2 k+1) \in \mathcal{W}_{(m, 3)}$, 由引理 4 可得 $t_{i} \notin \mathcal{S}((s-m-1,2 k+2) \rightarrow)$. 而 $(s-m, 2 k) \in \mathcal{S}((s-m-1,2 k+2) \rightarrow)$, 所以 $t_{2 k} \neq(s-m, 2 k)$. 由引理 1 和 5 知 $t_{2 k}$ 是 $(s-m, 2 k)$ 的 $\tau$ - 后继元, 在 $\mathcal{W}^{(0,1)}$ 中考察, 存在 $i \in\{2,4, \cdots, 2 k-2\}$, 使得 $t_{i} \in \mathcal{S}((s-m-1,2 k+2) \rightarrow)$, 矛盾, 所以 $t_{2 k+1} \notin \mathcal{W}_{(m, 3)}$.

对偶地讨论知 $t_{2 k+1} \notin \mathcal{W}_{(-m-1,3)}$, 所以 $t_{2 k+1}$ 与 $t_{2 k-1}$ 是邻点.

对偶地可知, 当 $i, j \leqslant 2 k+1$ 时命题成立, 则当 $i, j \leqslant 2 k+2$ 时命题也成立, 由数学归纳法, 当 $\Delta$ 为 $A_{2 m}$ 型时, 定理成立.

\section{$3.4 D_{n}(n \geqslant 4), E_{q}(q=6,7,8)$ 型情形}

下面考虑 $D_{n}$ 型的情形. 不妨考虑变换图 $Z \Delta_{D_{n}}$, 这里 $\Delta_{D_{n}}$ 为

$$
\begin{array}{r}
n \longleftarrow \\
n-1 \longleftarrow
\end{array} \longleftarrow 2 \rightarrow \cdots \rightarrow n-3 \rightarrow n-2 \text { (图 } 4 \text { 是 } n=7 \text { 的情形). }
$$




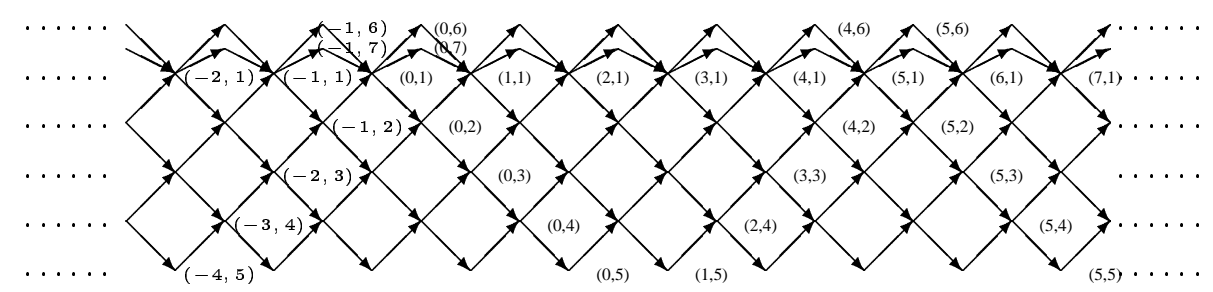

图 $4 \quad Z \Delta_{D_{7}}$

引理 6 在 $Z \Delta_{D_{n}}$ 中,

(1) 以 $(s, t)$ 为起点的 hammock 的终点为 $(s+n-2, t)$;

(2) ${ }_{x} H_{y}$ 是 hammock, 若 $x, y$ 是在 $(0,1)$ 所在的 $\tau$ - 轨道上, 则 ${ }_{x} H_{y}=\{z \mid \mathcal{S}(x$ $\rightarrow) \leqslant z \leqslant \mathcal{S}(\rightarrow y)\}$.

引理 7 设 $\Gamma_{A}$ 是 $D_{n}$ 型的路代数的 AR- 箭图, $\Gamma_{A}$ 嵌入 $Z \Delta_{D_{n}}$. 设 $(s, 1)$ 是 $\Gamma_{A}$ 中某个点的 $\tau$ - 后继元, $(s, t) \in \Gamma_{A}, t \leqslant n-2$, 则 $(s, 1) \rightarrow(s, 2) \rightarrow \cdots \rightarrow(s, t)$ 是 $\Gamma_{A}$ 的子图.

将 $\Gamma_{A}$ 作为 $Z \Delta_{D_{n}}$ 的子图. 不妨设 $T_{1}$ 对应 $(0,1), T_{i}$ 对应的点 $t_{i}$ 在 $(0, i)$ 所在 的 $\tau$ - 轨道上.

记 $\mathcal{W}(0,1)=\{z \mid \mathcal{S}(\rightarrow(0,1)) \leqslant z \leqslant \mathcal{S}((0,1) \rightarrow)\}, \mathcal{W}_{(n-2,3)}=\{z \mid \mathcal{S}((3, n-$ $2) \rightarrow) \leqslant z \leqslant \mathcal{S}(\rightarrow(n-2, n-2))\}, \mathcal{W}_{(-n, 3)}=\{z \mid \mathcal{S}((-(2 n-5), n-2) \rightarrow) \leqslant z \leqslant$ $\mathcal{S}(\rightarrow(-n, n-2))\}$. 记 $\overline{\mathcal{W}}=\mathcal{W}(0,1) \cup \mathcal{W}_{(n-2,3)} \bigcup \mathcal{W}_{(-n, 3)}$. 易证, $t_{i} \in \overline{\mathcal{W}}(1 \leqslant i \leqslant$ $n)$.

因为在 $\overline{\mathcal{W}}$ 中, $t_{1}=(0,1), t_{2} \in\{(-1,2),(0,2)\}, t_{n-1} \in\{(-1, n-1),(0, n-$ 1) $\}, t_{n} \in\{(-1, n),(0, n)\}$, 这样若 $t_{i}$ 与 $t_{j}(i, j \in\{1,2, n-1, n\})$ 的 $\tau$ - 轨道相邻, 则 $t_{i}$ 与 $t_{j}$ 是邻点.

假设当 $i, j \leqslant k \leqslant n-3$ 时, $t_{i}$ 与 $t_{j}$ 的 $\tau$ - 轨道相邻, 则 $t_{i}$ 与 $t_{j}$ 是邻点, 考虑 $t_{k+1}$. 如果 $t_{k+1}=(s, k+1) \in \mathcal{W}_{(n-2,3)}$, 由引理 6 可得 $t_{i} \notin \mathcal{S}((s-n+1, k+1) \rightarrow)$. 而 $(s-n+2, k) \in \mathcal{S}((s-n+1, k+1) \rightarrow)$, 所以 $t_{k} \neq(s-n+2, k)$. 由引理 $7,(s, 1) \rightarrow(s, 2) \rightarrow \cdots \rightarrow(s, k) \rightarrow(s, k+1)$ 是 $\Gamma_{A}$ 的子图. 由引理 1 知 $t_{k}$ 是 $(s-n+2, k)$ 的 $\tau$ - 后继元. 在 $\mathcal{W}(0,1)$ 中考察, 存在 $i \in\{1,2, \cdots, k-1\}$, 使得 $t_{i} \in \mathcal{S}((s-n+1, k+1) \rightarrow)$, 矛盾, 所以 $t_{k+1} \notin \mathcal{W}_{(n-2,3)}$.

对偶地讨论知 $t_{k+1} \notin \mathcal{W}_{(-n, 3)}$, 因而 $t_{k+1} \in \mathcal{W}_{(0,1)}$, 所以 $t_{k+1}$ 与 $t_{k}$ 是邻点. 当 $\Delta$ 为 $D_{n}(n \geqslant 4)$ 型时定理成立.

下面考虑 $E_{6}$ 型的情形. 不妨考虑变换图 $Z \Delta_{E_{6}}$, 这里 $\Delta_{E_{6}}$ 为

$$
\begin{aligned}
& 6 \longleftarrow \\
& 5 \longleftarrow 4 \longleftarrow
\end{aligned} \text { - } 2 \rightarrow 1
$$

引理 8 在图 5 中, 


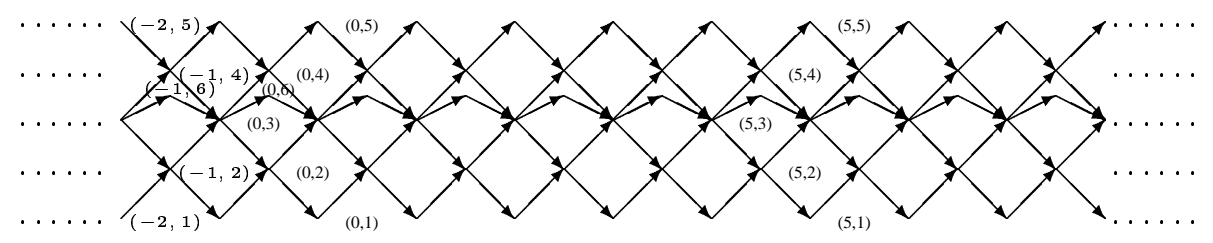

图 $5 Z \Delta_{E_{6}}$

(1) 以 $(s, t)$ 为起点的 hammock 的终点为

(a) $(s+5,6-t)$, 当 $t \neq 6$ 时;

(b) $(s+5,6)$, 当 $t=6$ 时.

(2) 设 ${ }_{x} H_{y}$ 是 hammock, 若 $x, y$ 是在 $(0,3)$ 所在的 $\tau$ - 轨道, 则

$$
{ }_{x} H_{y}=\{z \mid \mathcal{S}(x \rightarrow) \leqslant z \leqslant \mathcal{S}(\rightarrow y)\} .
$$

将 $\Gamma_{A}$ 作为 $Z \Delta_{E_{6}}$ 的子图. 不妨设 $T_{3}$ 对应 $(0,3), T_{i}$ 对应的点 $t_{i}$ 在 $(0, i)$ 所 在的 $\tau$ - 轨道上. 易证 $t_{i} \in \mathcal{W}(0,3) \bigcup\{(-7,1),(5,1),(-7,5),(5,5)\}$. 如果 $t_{1}=$ $(5,1)$, 则 $H_{t_{1}}$ 的起点为 $(0,5)$. 由引理 1 知, $\mathcal{S}(\rightarrow(0,5)) \leqslant t_{i}$, 所以 $t_{4}=(0,4)$. 这样 $\operatorname{Ext}_{A}^{1}\left(T_{1}, T_{4}\right)=D \operatorname{Hom}_{A}\left(T_{4}, \tau T_{1}\right) \neq 0$, 矛盾. 同样可证 $t_{1} \neq(-7,1), t_{5} \notin$ $\{(-7,5),(5,5)\}$, 即 $t_{i} \in \mathcal{W}(0,3)$. 所以命题成立，故当 $\Delta$ 为 $E_{6}$ 型时定理成立.

同理可证 $E_{7}, E_{8}$ 型的情形也成立.

\section{参考文献}

1 Happel D, Ringel C M. Tilted algebras. Trans Amer Math Soc, 1982, 274: 399 433

2 Ringel C M. Tame Algebra and Integral Quadratic Forms. LNM1099. New York: Springer-Verlag, 1984

3 章 璞. 分离倾斜模. 科学通报, 1991, 36(20): 1524 1526

4 Ringel C M, Vossieck D. Hammocks. Proc London Math Soc, 1987, 54(3): 216 246 